\title{
PRODUKTIONSKOSTEN DES SEKTORS STAAT
}

Die Entscheidungen über die Menge und Art der zu produzierenden Güter und Dienstleistungen ebenso wie über die beste Produktionsweise sind oft politischer Natur und basieren auf dem sozialen und kulturellen Kontext des jeweiligen Landes. Während einige Regierungen einen großen Teil der Produktion von Gütern und Dienstleistungen an nichtstaatliche bzw. private Einrichtungen auslagern, produzieren andere diese Güter und Dienstleistungen selbst.

\section{Definition}

Die staatlichen Güter und Dienstleistungen werden durch die Beschäftigten im öffentlichen Dienst, den Einsatz von Kapital sowie externe Anbieter (Einrichtungen ohne Erwerbszweck oder Einrichtungen des privaten Sektors) produziert. Letzteres wird oft als „Auslagerung“ bezeichnet.

Dieses Konzept und diese Methodik der Produktionskosten stützen sich auf die bestehende Klassifizierung der Staatsausgaben im System der Volkswirtschaftlichen Gesamtrechnungen (SNA). Konkret zählen zu den Kosten der staatlichen

\section{Überblick}

2010 machten die Produktionskosten staatlicher Güter und Dienstleistungen in den OECD-Ländern durchschnittlich nahezu ein Viertel des BIP aus, mit deutlichen Schwankungen zwischen den Ländern. So waren die Produktionskosten der staatlichen Güter und Dienstleistungen in Prozent des BIP z.B. in Dänemark etwa dreimal so hoch wie in Mexiko, was teilweise auf die unterschiedliche Rolle des Staats in diesen Ländern zurückzuführen ist. Im Durchschnitt ist die Produktion durch eigene Staatsbedienstete nach wie vor etwas stärker ausgeprägt als die Auslagerung: $48 \%$ der Produktionskosten für Güter und Dienstleistungen entfallen auf Arbeitnehmerentgelt, während $43 \%$ als Zahlung an nichtstaatliche Akteure anfallen, die Zwischenprodukte und Dienstleistungen produzieren oder Dienstleistungen direkt für private Haushalte erbringen. Die übrigen $9 \%$ der staatlichen Gesamtproduktionskosten entfallen auf die Abschreibung von Anlagevermögen. In den Niederlanden, Deutschland und Japan, wo über $55 \%$ des Werts der staatlichen Güter und Dienstleistungen ausgelagert werden, ist der Anteil der von Unternehmen und privaten Einrichtungen ohne Erwerbszweck produzierten Güter und Dienstleistungen im Vergleich höher als in anderen OECD-Mitgliedsländern.

Die Gesamtproduktionskosten des Sektors Staat im Verhältnis zum BIP sind zwischen 2001 und 2010 im OECDRaum mit Ausnahme von fünf OECD-Mitgliedsländern (Israel, Polen, die Slowakische Republik, Schweden und die Schweiz) angestiegen. Dieser Anstieg war hauptsächlich auf auslagerungsbezogene Kostensteigerungen (die Kosten der von nichtstaatlichen Anbietern produzierten Güter und Dienstleistungen stiegen um 1,4 Prozentpunkte) und in geringerem Umfang auf den Anstieg der Vergütungskosten für Beschäftigte im öffentlichen Dienst (0,5 Prozentpunkte) zurückzuführen. Diese Zuwächse könnten sich daraus ergeben, dass der Staat mehr Güter und Dienstleistungen bereitstellt und/oder dass die Vorleistungskosten gestiegen sind.
Produktion die folgenden: Vergütungskosten für die Beschäftigten des Sektors Staat, Kosten der von nichtstaatlichen Einrichtungen produzierten und vom Staat bezahlten Güter und Dienstleistungen (diese umfassen sowohl für den Staat als auch für die Bürger bereitgestellte Güter und Dienstleistungen) sowie Abschreibungen (nutzungsbedingte Wertminderungen).

Die Daten umfassen die Beschäftigung im öffentlichen Dienst und die Vorleistungen für Produkte, die vom Staat zur eigenen Verwendung produziert wurden, z.B. für von öffentlich Bediensteten gebaute Straßen und andere Anlageinvestitionsprojekte.

\section{Vergleichbarkeit}

Die Angaben basieren auf dem System der Volkswirtschaftlichen Gesamtrechnungen von 1993 (SNA 1993) bzw. dem Europäischen System Volkswirtschaftlicher Gesamtrechnungen von 1995 (ESVG 1995), was bedeutet, dass alle Länder den gleichen Definitionskatalog verwenden. Die Länderunterschiede bei der Finanzierung der Rentensysteme können den Vergleich der Vergütungskosten jedoch beeinträchtigen. Hinzukommt, dass die sozialen Sachtransfers über Marktproduzenten in einigen Ländern nicht getrennt ausgewiesen werden.

Die Daten zu Australien, Chile, Korea, Neuseeland und der Russischen Föderation beziehen sich auf 2009 anstelle von 2010. Die Daten für Mexiko beziehen sich auf 2003 anstelle von 2001. Die Daten für die Russische Föderation beziehen sich auf 2002 anstelle von 2001. Beim OECD-Durchschnitt der Produktionskosten in Prozent des BIP werden Chile, die Türkei und Japan nicht berücksichtigt.

\section{Quelle}

- OECD (2012), Government at a Glance, OECD Publishing.

\section{Weitere Informationen}

\section{Analysen}

- OECD (2012), Corporate Governance, Value Creation and Growth, The Bridge between Finance and Enterprise, Corporate Governance, OECD Publishing.

- OECD (2008), The State of the Public Service, OECD Publishing.

\section{Statistiken}

- OECD (2012), National Accounts at a Glance, OECD Publishing.

- OECD (2012), National Accounts of OECD Countries, OECD Publishing.

\section{Online-Datenbanken}

- "General Government Accounts: Main aggregates", OECD National Accounts Statistics.

\section{Websites}

- Government at a Glance (ergänzendes Material), www.oecd.org/gov/indicators/govataglance. 


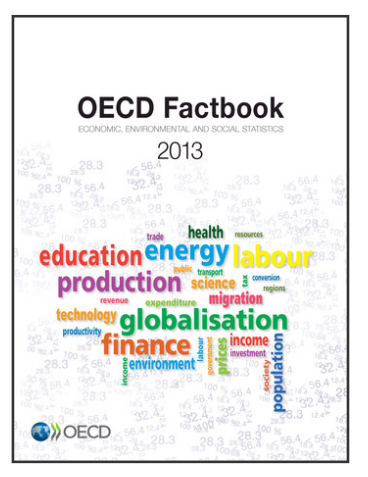

\section{From: \\ OECD Factbook 2013 \\ Economic, Environmental and Social Statistics}

Access the complete publication at:

https://doi.org/10.1787/factbook-2013-en

\section{Please cite this chapter as:}

OECD (2013), "Produktionskosten des Sektors Staat", in OECD Factbook 2013: Economic, Environmental and Social Statistics, OECD Publishing, Paris.

DOI: https://doi.org/10.1787/factbook-2013-85-de

Das vorliegende Dokument wird unter der Verantwortung des Generalsekretärs der OECD veröffentlicht. Die darin zum Ausdruck gebrachten Meinungen und Argumente spiegeln nicht zwangsläufig die offizielle Einstellung der OECDMitgliedstaaten wider.

This document and any map included herein are without prejudice to the status of or sovereignty over any territory, to the delimitation of international frontiers and boundaries and to the name of any territory, city or area.

You can copy, download or print OECD content for your own use, and you can include excerpts from OECD publications, databases and multimedia products in your own documents, presentations, blogs, websites and teaching materials, provided that suitable acknowledgment of OECD as source and copyright owner is given. All requests for public or commercial use and translation rights should be submitted to rights@oecd.org. Requests for permission to photocopy portions of this material for public or commercial use shall be addressed directly to the Copyright Clearance Center (CCC) at info@copyright.com or the Centre français d'exploitation du droit de copie (CFC) at contact@cfcopies.com. 